\title{
On some results in intuitionistic fuzzy ideal convergence double sequence spaces
}

\author{
Vakeel A. Khan ${ }^{1 *}$, Mobeen Ahmad ${ }^{1}$, Hira Fatima ${ }^{1}$ and Mohd Faisal Khan ${ }^{2}$
}

"Correspondence:

vakhanmaths@gmail.com

'Department of Mathematics,

Aligarh Muslim University, Aligarh,

India

Full list of author information is

available at the end of the article

\section{Springer}

\begin{abstract}
Recently, spaces of ideal convergent sequences of bounded linear operators were studied by Khan et al. (Numer. Funct. Anal. Optim. 39:1278-1290, 2018). This has motivated us to propose the intuitionistic fuzzy 1 -convergent double sequence spaces determined by the bounded linear operator. In this paper, we investigate the algebraic and topological properties. We also study the concept of the ideal Cauchy and ideal convergence on the said spaces.
\end{abstract}

Keywords: Bounded linear operator; Intuitionistic fuzzy normed spaces; Ideal; Filter; I-convergence

\section{Introduction}

Zadeh [27] introduced the concept of fuzzy sets in 1965 and Goguen [6] extended it to Lfuzzy sets. As far as the theme of the concept of fuzzy sets is concerned, the idea has been utilized by the researchers around the globe heavily. Fuzzy sets have been put on to the metric spaces and emerged as fuzzy metric spaces studied by George et al. [5] and Amini et al. [1]. Furthermore, the idea of $I$ and $I^{*}$ convergent sequences in fuzzy normed spaces was due to Kumar et al. [14]. In 1986, Atanassov [2] started the study of intuitionistic fuzzy sets which is a generalization of fuzzy sets. Park [20] initiated the notion of intuitionistic fuzzy metric spaces moreover, Saadati et al. [21] extended this idea to intuitionistic fuzzy topological spaces. The study of the convergence of sequences in a fuzzy normed space is vital to fuzzy functional analysis, we feel that $I$-convergence in intuitionistic fuzzy normed space would yield a more general foundation. Later on, statistical convergence and ideal convergence of sequences concerning intuitionistic fuzzy normed space were studied by Mursaleen et al. [16, 19]. Moreover, the contributions to the study of intuitionistic fuzzy metric spaces and intuitionistic fuzzy normed spaces can be found in $[7,10,11,24]$.

The notion of ideal convergence was initiated by Kostyrko et al. [12] using the concept of the ideal $I$ as a subset of the set of positive integers which is a generalization of statistical convergence given by Fast [4] in 1951. Furthermore, it was examined from the sequence space viewpoint and connected with the summability theory by Šalát et al. [22, 23]; Tripathy et al. [25, 26] defined paranorm I-convergent sequence spaces; Khan et al. [9] studied the ideal convergent sequence of bounded linear operator. Later on, Das et al. [3] studied $I$ and $I^{*}$-convergence of double sequences. Mursaleen et al. $[15,17,18]$ analyzed ideal convergence in random 2-normed spaces and probabilistic normed spaces.

(c) The Author(s) 2019. This article is distributed under the terms of the Creative Commons Attribution 4.0 International License (http://creativecommons.org/licenses/by/4.0/), which permits unrestricted use, distribution, and reproduction in any medium, provided you give appropriate credit to the original author(s) and the source, provide a link to the Creative Commons license, and indicate if changes were made. 
Our aim for the present paper is to discuss the concept of intuitionistic fuzzy ideal convergence of double sequence spaces defined by the bounded linear operator which would yield a more convenient structure to deal with the inexactness of the sequence spaces in some situations.

\section{Preliminaries}

Now, we present some notations and basic definitions.

Definition 2.1 ([12]) A family of sets $I \subseteq 2^{Y}$ is called an ideal in nonempty set $Y$, if

- $\emptyset \in I$

- $I$ is additive; that is, $C, D \in I \Rightarrow C \cup D \in I$;

- $I$ is hereditary that is, $C \in I, D \subseteq C \Rightarrow D \in I$.

$I \subseteq 2^{Y}$ is said to be nontrivial if $I \neq 2^{Y}$. If $\{\{y\}: y \in Y\} \subseteq I$, then a nontrivial ideal $I \subseteq 2^{Y}$ is called admissible. $I$ is maximal if there cannot exist any nontrivial ideal $J$ containing $I$ as a subset.

Definition 2.2 ([12]) Suppose $Y$ is a nonempty set. Then $\mathcal{F} \subset 2^{Y}$ is called a filter on $Y$ if and only if the following implications hold:

- $\emptyset \notin \mathcal{F}$;

- for $C, D \in \mathcal{F} \Rightarrow C \cap D \in \mathcal{F}$;

- for each $C \in \mathcal{F}$ and $D \supset C \Rightarrow D \in \mathcal{F}$.

For every ideal $I$ there corresponds a filter defined as

$$
\mathcal{F}(I)=\left\{K \subset Y: K^{c} \in I \text {, where } K^{c}=Y-K\right\} .
$$

We take $I_{2}$ as a nontrivial ideal in $\mathbb{N} \times \mathbb{N}$ throughout the paper.

Definition 2.3 ([26]) A double sequence $y=\left(y_{i j}\right) \in{ }_{2} \omega$ is said to be $I_{2}$-convergent to $L$ if, for every $\epsilon>0$, we have

$$
\left\{(i, j) \in \mathbb{N} \times \mathbb{N}:\left|y_{i j}-L\right| \geq \epsilon\right\} \in I_{2}
$$

We write $I_{2}-\lim y_{i j}=L$.

Definition 2.4 ([26]) A sequence $y=\left(y_{i j}\right)$ is said to be $I_{2}$-Cauchy, if for each $\epsilon>0$, there exist positive integers $m=m(\epsilon)$ and $n=n(\epsilon)$ such that the set

$$
\left\{(i, j) \in \mathbb{N} \times \mathbb{N}:\left|y_{i j}-y_{m n}\right| \geq \epsilon\right\} \in I_{2} .
$$

Definition 2.5 ([19]) Let $(Y, \phi, \psi, *, \diamond)$ be an intuitionistic fuzzy normed space (IFNS). A sequence $y=\left(y_{i j}\right)$ is termed convergent to $L \in Y$ under intuitionistic fuzzy norm $(\phi, \psi)$ if, for every $\epsilon, t>0$, there exist $k_{0} \in \mathbb{N}$ such that $\phi\left(y_{i j}-L, t\right)>1-\epsilon$ and $\psi\left(y_{i j}-L, t\right)<\epsilon$ for all $i, j \geq k_{0}$.

Definition 2.6 ([19]) Let $(Y, \phi, \psi, *, \diamond)$ be an IFNS. A sequence $y=\left(y_{i j}\right)$ is termed a Cauchy sequence with respect to the intuitionistic fuzzy norm $(\phi, \psi)$, if for every $\epsilon, t>0, \exists k_{0} \in \mathbb{N}$ such that $\phi\left(y_{i j}-y_{m n}, t\right)>1-\epsilon$ and $\psi\left(y_{i j}-y_{m n}, t\right)<\epsilon$, for all $i, j, m, n \geq k_{0}$. 
Remark ([21]) If $*$ is a continuous $t$-norm, $\diamond$ is a continuous $t$-conorm and $p_{i} \in(0,1)$, $1 \leq i \leq 7$. Then:

- for any $p_{1}, p_{2} \in(0,1)$ with $p_{1}>p_{2}$, there exist $p_{3}, p_{4} \in(0,1)$ such that

$$
p_{1} * p_{3} \geq p_{2} \quad \text { and } \quad p_{1} \geq p_{4} \diamond p_{2}
$$

- for any $p_{5} \in(0,1)$, there exist $p_{6}, p_{7} \in(0,1)$ such that $p_{6} * p_{6} \geq p_{5}$ and $p_{7} \diamond p_{7} \leq p_{5}$.

Definition 2.7 ([19]) Let $I_{2} \subset 2^{\mathbb{N} \times \mathbb{N}}$ be a nontrivial ideal and $(Y, \phi, \psi, *, \diamond)$ be an IFNS. A sequence $y=\left(y_{i j}\right)$ in $Y$ is called $I_{2}$-convergent to $L \in Y$ with respect to the intuitionistic fuzzy norm $(\phi, \psi)$ if, for every $\epsilon, t>0$, the set

$$
\left\{(i, j): \phi\left(y_{i j}-L, t\right) \leq 1-\epsilon \text { or } \psi\left(y_{i j}-L, t\right) \geq \epsilon\right\} \in I_{2} .
$$

We write $I_{2}^{(\phi, \psi)}-\lim y_{i j}=L$.

Definition 2.8 ([19]) Let $(Y, \phi, \psi, *, \diamond)$ be an IFNS. A sequence $y=\left(y_{i j}\right)$ in $Y$ is called an $I_{2}$-Cauchy sequence with respect to the intuitionistic fuzzy norm $(\phi, \psi)$, if, for every $\epsilon$, $t>0$, the set

$$
\left\{(i, j): \phi\left(y_{i j}-y_{m n}, t\right) \leq 1-\epsilon \text { or } \psi\left(y_{i j}-y_{m n}, t\right) \geq \epsilon\right\} \in I_{2} .
$$

Definition 2.9 ([13]) Let $U$ and $V$ be two normed linear spaces and $B: \mathcal{D}(B) \rightarrow V$ be a linear operator, where $\mathcal{D}(B) \subset U$. An operator $B$ is bounded, if there exists $k>0$ such that

$$
\|B x\| \leq k\|x\|, \quad \text { for all } x \in \mathcal{D}(B) .
$$

We denote by $\mathcal{B}(U, V)$ the set of all bounded linear operators which is normed linear spaces normed by

$$
\|B\|=\sup _{x \in U,\|x\|=1}\|B x\|
$$

and $\mathcal{B}(U, V)$ is a Banach space if $V$ is a Banach space.

\section{Main results}

In this section, we introduce the following new sequence spaces:

$$
\begin{aligned}
& { }_{2} S_{(\phi, \psi)}^{I_{2}}(B)=\left\{\left(x_{i j}\right) \in{ }_{2} \ell_{\infty}:\left\{(i, j): \phi\left(B\left(x_{i j}\right)-L, t\right) \leq 1-\epsilon \text { or } \psi\left(B\left(x_{i j}\right)-L, t\right) \geq \epsilon\right\} \in I_{2}\right\} ; \\
& { }_{2} S_{0(\phi, \psi)}^{I_{2}}(B)=\left\{\left(x_{i j}\right) \in{ }_{2} \ell_{\infty}:\left\{(i, j): \phi\left(B\left(x_{i j}\right), t\right) \leq 1-\epsilon \text { or } \psi\left(B\left(x_{i j}\right), t\right) \geq \epsilon\right\} \in I_{2}\right\} .
\end{aligned}
$$

An open ball with center $x$ and radius $r$ with respect to $t$ is defined as

$$
{ }_{2} \mathcal{B}_{x}(r, t)(B)=\left\{\left(y_{i j}\right) \in{ }_{2} \ell_{\infty}: \phi\left(B\left(x_{i j}\right)-B\left(y_{i j}\right), t\right)>1-r \text { and } \psi\left(B\left(x_{i j}\right)-B\left(y_{i j}\right), t\right)<r\right\} .
$$

Theorem $3.1{ }_{2} S_{(\phi, \psi)}^{I_{2}}(B)$ and ${ }_{2} S_{0(\phi, \psi)}^{I_{2}}(B)$ are linear spaces. 
Proof Let $x=\left(x_{i j}\right), y=\left(y_{i j}\right) \in{ }_{2} S_{(\phi, \psi)}^{I_{2}}(B)$ and $\alpha, \beta$ be scalars. For a given $\epsilon>0$, we obtain

$$
\begin{aligned}
& A_{1}=\left\{(i, j): \phi\left(B\left(x_{i j}\right)-L_{1}, \frac{t}{2|\alpha|}\right) \leq 1-\epsilon \text { and } \psi\left(B\left(x_{i j}\right)-L_{1}, \frac{t}{2|\alpha|}\right) \geq \epsilon\right\} \in I_{2} ; \\
& A_{2}=\left\{(i, j): \phi\left(B\left(y_{i j}\right)-L_{2}, \frac{t}{2|\beta|}\right) \leq 1-\epsilon \text { and } \psi\left(B\left(y_{i j}\right)-L_{2}, \frac{t}{2|\beta|}\right) \geq \epsilon\right\} \in I_{2} . \\
& A_{1}^{c}=\left\{(i, j): \phi\left(B\left(x_{i j}\right)-L_{1}, \frac{t}{2|\alpha|}\right)>1-\epsilon \text { and } \psi\left(B\left(x_{i j}\right)-L_{1}, \frac{t}{2|\alpha|}\right)<\epsilon\right\} \in \mathcal{F}\left(I_{2}\right) ; \\
& A_{2}^{c}=\left\{(i, j): \phi\left(B\left(y_{i j}\right)-L_{2}, \frac{t}{2|\beta|}\right)>1-\epsilon \text { and } \psi\left(B\left(y_{i j}\right)-L_{2}, \frac{t}{2|\beta|}\right)<\epsilon\right\} \in \mathcal{F}\left(I_{2}\right) .
\end{aligned}
$$

Define $A_{3}=A_{1} \cup A_{2}$, so that $A_{3} \in I_{2}$. It implies that $A_{3}^{c}$ is a nonempty set in $\mathcal{F}\left(I_{2}\right)$. Now, we have to show that, for each $\left(x_{i j}\right),\left(y_{i j}\right) \in{ }_{2} S_{(\phi, \psi)}^{I_{2}}(B), A_{3}^{c} \subset\left\{(i, j): \phi\left(\left(\alpha B\left(x_{i j}\right)+\beta B\left(y_{i j}\right)\right)-\left(\alpha L_{1}+\right.\right.\right.$ $\left.\left.\beta L_{2}\right), t\right)>1-\epsilon$ and $\left.\psi\left(\left(\alpha B\left(x_{i j}\right)+\beta B\left(y_{i j}\right)\right)-\left(\alpha L_{1}+\beta L_{2}\right), t\right)<\epsilon\right\}$. Let $m, n \in A_{3}^{c}$. In this case

$$
\begin{aligned}
& \phi\left(B\left(x_{m n}\right)-L_{1}, \frac{t}{2|\alpha|}\right)>1-\epsilon \text { and } \psi\left(B\left(x_{m n}\right)-L_{1}, \frac{t}{2|\alpha|}\right)<\epsilon, \\
& \phi\left(B\left(y_{m n}\right)-L_{2}, \frac{t}{2|\beta|}\right)>1-\epsilon \text { and } \psi\left(B\left(y_{m n}\right)-L_{2}, \frac{t}{2|\beta|}\right)<\epsilon .
\end{aligned}
$$

We have

$$
\begin{aligned}
& \phi\left(\left(\alpha B\left(x_{m n}\right)+\beta B\left(y_{m n}\right)\right)-\left(\alpha L_{1}+\beta L_{2}\right), t\right) \\
& \geq \phi\left(\alpha B\left(x_{m n}\right)-\alpha L_{1}, \frac{t}{2}\right) \\
& \quad * \phi\left(\beta B\left(y_{m n}\right)-\beta L_{2}, \frac{t}{2}\right) \\
& \geq \phi\left(B\left(x_{m n}\right)-L_{1}, \frac{t}{2|\alpha|}\right) * \phi\left(B\left(y_{m n}\right)-L_{2}, \frac{t}{2|\beta|}\right) \\
& \geq(1-\epsilon) *(1-\epsilon) \\
& >1-\epsilon .
\end{aligned}
$$

Also

$$
\begin{aligned}
\psi\left(\left(\alpha B\left(x_{m n}\right)+\beta B\left(y_{m n}\right)\right)-\left(\alpha L_{1}+\beta L_{2}\right), t\right) & \leq \psi\left(\alpha B\left(x_{m n}\right)-\alpha L_{1}, \frac{t}{2}\right) \\
& \diamond \psi\left(\beta B\left(y_{m n}\right)-\beta L_{2}, \frac{t}{2}\right) \\
& \leq \psi\left(B\left(x_{m n}\right)-L_{1}, \frac{t}{2|\alpha|}\right) \\
& \diamond \psi\left(B\left(y_{m n}\right)-L_{2}, \frac{t}{2|\beta|}\right) \\
& \leq \epsilon \diamond \\
& <\epsilon .
\end{aligned}
$$


This implies

$$
\begin{gathered}
A_{3}^{c} \subset\left\{(i, j): \phi\left(\left(\alpha B\left(x_{i j}\right)+\beta B\left(y_{i j}\right)\right)-\left(\alpha L_{1}+\beta L_{2}\right), t\right)>1-\epsilon\right. \\
\text { and } \left.\psi\left(\left(\alpha B\left(x_{i j}\right)+\beta B\left(y_{i j}\right)\right)-\left(\alpha L_{1}+\beta L_{2}\right), t\right)<\epsilon\right\} .
\end{gathered}
$$

Hence ${ }_{2} S_{(\phi, \psi)}^{I_{2}}(B)$ is a linear space.

In a similar way, we can prove that ${ }_{2} S_{0(\phi, \psi)}^{I_{2}}(B)$ is linear space.

Theorem 3.2 Every open ball ${ }_{2} \mathcal{B}_{x}(r, t)(B)$ is an open set in ${ }_{2} S_{(\phi, \psi)}^{I_{2}}(B)$.

Proof Suppose $y \in{ }_{2} \mathcal{B}_{x}(r, t)(B)$. Then, from the definition of ${ }_{2} \mathcal{B}_{x}(r, t)(B)$, we have

$$
\phi\left(B\left(x_{i j}\right)-B\left(y_{i j}\right), t\right)>1-r \quad \text { and } \quad \psi\left(B\left(x_{i j}\right)-B\left(y_{i j}\right), t\right)<r .
$$

From (3.1), there exists $t_{0} \in(0, t)$ such that $\phi\left(B\left(x_{i j}\right)-B\left(y_{i j}\right), t_{0}\right)>1-r$ and $\psi\left(B\left(x_{i j}\right)-\right.$ $\left.B\left(y_{i j}\right), t_{0}\right)<r$. Setting $p_{0}=\phi\left(B\left(x_{i j}\right)-B\left(y_{i j}\right), t_{0}\right)$. This implies that $p_{0}>1-r$. Thus, there exists $s \in(0,1)$ with $p_{0}>1-s>1-r$. For given $p_{0}, s$ with $p_{0}>1-s$, there exist $p_{1}, p_{2} \in(0,1)$ such that $p_{0} * p_{1}>1-s$ and $\left(1-p_{0}\right) \diamond\left(1-p_{2}\right) \leq s$. Select $p_{3}=\max \left\{p_{1}, p_{2}\right\}$ and consider the ball ${ }_{2} \mathcal{B}_{y}\left(1-p_{3}, t-t_{0}\right)(B)$. Now, we show

$$
{ }_{2} \mathcal{B}_{y}\left(1-p_{3}, t-t_{0}\right)(B) \subset{ }_{2} \mathcal{B}_{x}(r, t)(B)
$$

Let $z=\left(z_{i j}\right) \in{ }_{2} \mathcal{B}_{y}\left(1-p_{3}, t-t_{0}\right)(B)$. Then $\phi\left(B\left(y_{i j}\right)-B\left(z_{i j}\right), t-t_{0}\right)>p_{3}$ and $\psi\left(B\left(y_{i j}\right)-B\left(z_{i j}\right), t-\right.$ $\left.t_{0}\right)<1-p_{3}$.

Therefore

$$
\begin{aligned}
\phi\left(B\left(x_{i j}\right)-B\left(z_{i j}\right), t\right) & \geq \phi\left(B\left(x_{i j}\right)-B\left(y_{i j}\right), t_{0}\right) * \phi\left(B\left(y_{i j}\right)-B\left(z_{i j}\right), t-t_{0}\right) \\
& \geq\left(p_{0} * p_{3}\right) \\
& \geq\left(p_{0} * p_{1}\right) \\
& \geq(1-s)>1-r
\end{aligned}
$$

and

$$
\begin{aligned}
\psi\left(B\left(x_{i j}\right)-B\left(z_{i j}\right), t\right) & \leq \psi\left(B\left(x_{i j}\right)-B\left(y_{i j}\right), t_{0}\right) \diamond \psi\left(B\left(y_{i j}\right)-B\left(z_{i j}\right), t-t_{0}\right) \\
& \leq\left(1-p_{0}\right) \diamond\left(1-p_{3}\right) \\
& \leq\left(1-p_{0}\right) \diamond\left(1-p_{2}\right) \\
& \leq s<r .
\end{aligned}
$$

The above inequalities imply that $z \in{ }_{2} \mathcal{B}_{x}(r, t)(B)$. Thus ${ }_{2} \mathcal{B}_{y}\left(1-p_{3}, t-t_{0}\right)(B) \subset$ ${ }_{2} \mathcal{B}_{x}(r, t)(B)$.

Remark Let ${ }_{2} S_{(\phi, \psi)}^{I_{2}}(B)$ be an IFNS. Define ${ }_{2} \tau_{(\phi, \psi)}^{I_{2}}(B)=\left\{K \subset{ }_{2} S_{(\phi, \psi)}^{I_{2}}(B)\right.$ : for each $x \in$ $K, \exists t>0$ and $r \in(0,1)$ such that $\left.{ }_{2} \mathcal{B}_{x}(r, t)(B) \subset K\right\}$. Then ${ }_{2} \tau_{(\phi, \psi)}^{I}(B)$ is a topology on ${ }_{2} S_{(\phi, \psi)}^{I_{2}}(B)$. 
Theorem 3.3 The spaces ${ }_{2} S_{(\phi, \psi)}^{I_{2}}(B)$ and ${ }_{2} S_{0(\phi, \psi)}^{I_{2}}(B)$ are Hausdorff.

Proof Let $x, y \in{ }_{2} S_{(\phi, \psi)}^{I_{2}}(B)$ be two distinct points. Then $0<\phi(B(x)-B(y), t)<1$ and $0<\psi(B(x)-B(y), t)<1$. Putting $p_{1}=\phi(B(x)-B(y), t), p_{2}=\psi(B(x)-B(y), t)$ and $p=$ $\max \left\{p_{1}, 1-p_{2}\right\}$.

For each $p_{0} \in(p, 1)$ there exist $p_{3}$ and $p_{4}$ such that $p_{3} * p_{3} \geq p_{0}$ and $\left(1-p_{4}\right) \diamond\left(1-p_{4}\right) \leq$ $\left(1-p_{0}\right)$.

Put $p_{5}=\max \left\{p_{3}, p_{4}\right\}$ and consider the open balls ${ }_{2} \mathcal{B}_{x}\left(1-r_{p}, \frac{t}{2}\right)$ and ${ }_{2} \mathcal{B}_{y}\left(1-p_{5}, \frac{t}{2}\right)$.

Clearly ${ }_{2} \mathcal{B}_{x}\left(1-p_{5}, \frac{t}{2}\right) \cap{ }_{2} \mathcal{B}_{y}\left(1-p_{5}, \frac{t}{2}\right)=\emptyset$.

If there exists $z \in{ }_{2} \mathcal{B}_{x}\left(1-p_{5}, \frac{t}{2}\right) \cap{ }_{2} \mathcal{B}_{y}\left(1-p_{5}, \frac{t}{2}\right)$, then

$$
\begin{aligned}
p_{1} & =\phi(B(x)-B(y), t) \\
& \geq \phi\left(B(x)-B(z), \frac{t}{2}\right) * \phi\left(B(z)-B(y), \frac{t}{2}\right) \\
& \geq p_{5} * p_{5} \\
& \geq p_{3} * p_{3} \geq p_{0}>p_{1}
\end{aligned}
$$

and

$$
\begin{aligned}
p_{2} & =\psi(B(x)-B(y), t) \\
& \leq \psi\left(B(x)-B(z), \frac{t}{2}\right) \diamond \psi\left(B(z)-B(y), \frac{t}{2}\right) \\
& \leq\left(1-p_{5}\right) \diamond\left(1-p_{5}\right) \\
& \leq\left(1-p_{4}\right) \diamond\left(1-p_{4}\right) \\
& \leq\left(1-p_{0}\right)<p_{2},
\end{aligned}
$$

which is a contradiction. Hence ${ }_{2} S_{(\phi, \psi)}^{I_{2}}(B)$ is Hausdorff space. Similarly, one can show that ${ }_{2} S_{0(\phi, \psi)}^{I_{2}}(B)$ is Hausdorff space.

Theorem $3.4{ }_{2} S_{(\phi, \psi)}^{I_{2}}(B)$ is an IFNS and ${ }_{2} \tau_{(\phi, \psi)}^{I_{2}}(B)$ is a topology on ${ }_{2} S_{(\phi, \psi)}^{I_{2}}(B)$. Then a sequence $\left(x_{i j}\right) \in{ }_{2} S_{(\phi, \psi)}^{I_{2}}(B), x_{i j} \rightarrow x$ if and only if $\phi\left(B\left(x_{i j}\right)-B(x), t\right) \rightarrow 1$ and $\psi\left(B\left(x_{i j}\right)-\right.$ $B(x), t) \rightarrow 0$ as $i, j \rightarrow \infty$.

Proof Choose $t_{0}>0$. Suppose $x_{i j} \rightarrow x$. Then for $0<r<1, \exists N_{0} \in \mathbb{N}$ such that $\left(x_{i j}\right) \in$ ${ }_{2} S_{(\phi, \psi)}^{I_{2}}(B)$ for all $i, j \geq N_{0}$,

$$
\begin{aligned}
{ }_{2} S_{(\phi, \psi)}^{I_{2}}(B)= & \left\{\left(x_{i j}\right) \in{ }_{2} \ell_{\infty}:\left\{(i, j): \phi\left(B\left(x_{i j}\right)-B(x), t\right) \leq 1-\epsilon \text { or } \psi\left(B\left(x_{i j}\right)-B(x), t\right) \geq \epsilon\right\}\right. \\
& \left.\in I_{2}\right\}
\end{aligned}
$$

such that ${ }_{2} S_{(\phi, \psi)}^{I_{2}}(B) \in \mathcal{F}\left(I_{2}\right)$. Then $1-\phi\left(B\left(x_{i j}\right)-B(x), t\right)<r$ and $\psi\left(B\left(x_{i j}\right)-B(x), t\right)<r$.

Hence $\phi\left(B\left(x_{i j}\right)-B(x), t\right) \rightarrow 1$ and $\psi\left(B\left(x_{i j}\right)-B(x), t\right) \rightarrow 0$ as $i, j \rightarrow \infty$.

On the other way around, if for each $t>0, \phi\left(B\left(x_{i j}\right)-B(x), t\right) \rightarrow 1$ and $\psi\left(B\left(x_{i j}\right)-B(x), t\right) \rightarrow$ 0 as $i, j \rightarrow \infty$. Then for $0<r<1, \exists N_{0} \in \mathbb{N}$ such that $1-\phi\left(B\left(x_{i j}\right)-B(x), t\right)<r$ and $\psi\left(B\left(x_{i j}\right)-\right.$ $B(x), t)<r$, for all $i, j \geq N_{0}$. This implies that $\phi\left(B\left(x_{i j}\right)-B(x), t\right)>1-r$ and $\psi\left(B\left(x_{i j}\right)-B(x), t\right)<$ $r$ for all $i, j \geq N_{0}$. Thus $\left(x_{i j}\right) \in{ }_{2} S_{(\phi, \psi)}^{I_{2}}(B)$ for all $i, j \geq N_{0}$ and hence $x_{i j} \rightarrow x$. 
Theorem 3.5 Let ${ }_{2} S_{(\phi, \psi)}^{I_{2}}(B)$ be an IFNS. If a double sequence $x=\left(x_{i j}\right)$ is $I_{2}$-convergent with respect to the intuitionistic fuzzy norms $(\phi, \psi)$, then the $I_{2}^{(\phi, \psi)}$-limit is unique.

Proof Let $I_{2}^{(\phi, \psi)}-\lim x=L_{1}$ and $I_{2}^{(\phi, \psi)}-\lim x=L_{2}$. For a given $\epsilon>0$, select $s>0$ in such a way that $(1-s) *(1-s)>1-\epsilon$ and $s \diamond s<\epsilon$. Then, for any $t>0$, we define the following sets:

$$
\begin{aligned}
& P_{\phi, 1}(s, t)=\left\{(i, j) \in \mathbb{N} \times \mathbb{N}: \phi\left(B\left(x_{i j}\right)-L_{1}, \frac{t}{2}\right) \leq 1-s\right\}, \\
& P_{\phi, 2}(s, t)=\left\{(i, j) \in \mathbb{N} \times \mathbb{N}: \phi\left(B\left(x_{i j}\right)-L_{2}, \frac{t}{2}\right) \leq 1-s\right\}, \\
& P_{\psi, 1}(s, t)=\left\{(i, j) \in \mathbb{N} \times \mathbb{N}: \psi\left(B\left(x_{i j}\right)-L_{1}, \frac{t}{2}\right) \geq s\right\}, \\
& P_{\psi, 2}(s, t)=\left\{(i, j) \in \mathbb{N} \times \mathbb{N}: \psi\left(B\left(x_{i j}\right)-L_{2}, \frac{t}{2}\right) \geq s\right\} .
\end{aligned}
$$

Since $I_{2}^{(\phi, \psi)}-\lim x=L_{1}$, we obtain $P_{\phi, 1}(s, t)$ and $P_{\psi, 1}(s, t) \in I_{2}$.

Moreover, using $I_{2}^{(\phi, \psi)}-\lim x=L_{2}$, we have $P_{\phi, 2}(s, t)$ and $P_{\psi, 2}(s, t) \in I_{2}$. Now, suppose that

$$
P_{\phi, \psi}(s, t)=\left(P_{\phi, 1}(s, t) \cup P_{\phi, 2}(s, t)\right) \cap\left(_{\psi, 1}(s, t) \cup P_{\psi, 2}(s, t)\right) \in I_{2} .
$$

Thus, $P_{\phi, \psi}(s, t) \in I_{2}$, implies that $P_{\phi, \psi}^{c}(s, t)$ is a nonempty set in $\mathcal{F}\left(I_{2}\right)$.

If $(i, j) \in P_{\phi, \psi}^{c}(s, t)$, then two possibilities arise:

$$
(i, j) \in P_{\phi, 1}^{c}(s, t) \cap P_{\phi, 2}(s, t) \quad \text { or } \quad(i, j) \in P_{\psi, 1}^{c}(s, t) \cap P_{\psi, 2}(s, t) .
$$

Firstly, we consider that $(i, j) \in P_{\phi, 1}^{c}(s, t) \cap P_{\phi, 2}^{c}(s, t)$. Then we get

$$
\phi\left(L_{1}-L_{2}, t\right) \geq \phi\left(B\left(x_{i j}\right)-L_{1}, \frac{t}{2}\right) * \phi\left(B\left(x_{i j}\right)-L_{2}, \frac{t}{2}\right)>(1-s) *(1-s)>1-\epsilon .
$$

Since $\epsilon>0$ was arbitrary, we obtain $\phi\left(L_{1}-L_{2}, t\right)=1$ for every $t>0$, which yields $L_{1}=L_{2}$. Under other conditions, if $(i, j) \in P_{\psi, 1}^{c}(s, t) \cap P_{\psi, 2}^{c}(s, t)$, then we may write

$$
\psi\left(L_{1}-L_{2}, t\right) \leq \psi\left(B\left(x_{i j}\right)-L_{1}, \frac{t}{2}\right) \diamond \psi\left(B\left(x_{i j}\right)-L_{2}, \frac{t}{2}\right)<s \diamond s<\epsilon .
$$

Therefore, we obtain $\psi\left(L_{1}-L_{2}, t\right)=0$, for all $t>0$, which yields $L_{1}=L_{2}$. Hence, in all cases, we find that the $I_{2}^{(\phi, \psi)}$-limit is unique.

Theorem 3.6 A sequence $x=\left(x_{i j}\right) \in{ }_{2} S_{(\phi, \psi)}^{I_{2}}(B)$ is $I_{2}$-convergent with respect to the intuitionistic fuzzy norm $(\phi, \psi)$ if and only if it is $I_{2}$-Cauchy with respect to same norm.

Proof Suppose that the sequence $x=\left(x_{i j}\right) \in{ }_{2} S_{(\phi, \psi)}^{I_{2}}(B)$ is $I_{2}$-convergent, i.e., $I_{2}^{(\phi, \psi)}$ $\lim x=L$. Take $s>0$, in such a way that $(1-s) *(1-s)>1-\epsilon$ and $s \diamond s<\epsilon$. For all $t>0$, we get

$$
P=\left\{(i, j) \in \mathbb{N} \times \mathbb{N}: \phi\left(B\left(x_{i j}\right)-L, t\right) \leq 1-s \text { or } \psi\left(B\left(x_{i j}\right)-L, t\right) \geq s\right\} \in I_{2} .
$$


This implies $P^{c}=\left\{(i, j) \in \mathbb{N} \times \mathbb{N}: \phi\left(B\left(x_{i j}\right)-L, t\right)>1-s\right.$ and $\left.\psi\left(B\left(x_{i j}\right)-L, t\right)<s\right\} \in \mathcal{F}\left(I_{2}\right)$. Suppose $(m, n) \in P^{c}$. Then we obtain

$$
\phi\left(B\left(x_{m n}\right)-L, t\right)>1-s \text { and } \psi\left(B\left(x_{m n}\right)-L, t\right)<s .
$$

Let $Q=\left\{(i, j) \in \mathbb{N} \times \mathbb{N}: \phi\left(B\left(x_{i j}\right)-B\left(x_{m n}\right), t\right) \leq 1-\epsilon\right.$ or $\left.\psi\left(B\left(x_{i j}\right)-B\left(x_{m n}\right), t\right) \geq \epsilon\right\}$.

Furthermore, we prove the inclusion $Q \subset P$. Let $(i, j) \in Q$, we have

$$
\phi\left(B\left(x_{i j}\right)-B\left(x_{m n}\right), \frac{t}{2}\right) \leq 1-\epsilon \quad \text { and } \quad \psi\left(B\left(x_{i j}\right)-B\left(x_{m n}\right), \frac{t}{2}\right) \geq \epsilon .
$$

There are two possible cases, firstly we consider $\phi\left(B\left(x_{i j}\right)-B\left(x_{m n}\right), t\right) \leq 1-\epsilon$. Then we have $\phi\left(B\left(x_{i j}\right)-L, \frac{t}{2}\right) \leq 1-s$, therefore $(i, j) \in P$. On the other hand, if $\phi\left(B\left(x_{i j}\right)-L, \frac{t}{2}\right)>1-s$ then

$$
\begin{aligned}
1-\epsilon & \geq \phi\left(B\left(x_{i j}\right)-B\left(x_{m n}\right), t\right) \\
& \geq \phi\left(B\left(x_{i j}\right)-L, \frac{t}{2}\right) * \phi\left(B\left(x_{m n}\right)-L, \frac{t}{2}\right)>(1-s) *(1-s)>1-\epsilon,
\end{aligned}
$$

which is impossible. Hence $Q \subset P$.

Similarly, consider $\psi\left(B\left(x_{i j}\right)-B\left(x_{m n}\right), t\right) \geq \epsilon$. Then we have $\psi\left(B\left(x_{i j}\right)-L, \frac{t}{2}\right) \geq s$, hence $(i, j) \in P$. Otherwise, if $\psi\left(B\left(x_{i j}\right)-L, \frac{t}{2}\right)<s$, then

$$
\epsilon \leq \psi\left(B\left(x_{i j}\right)-B\left(x_{m n}\right), t\right) \leq \psi\left(B\left(x_{i j}\right)-L, \frac{t}{2}\right) \diamond \psi\left(B\left(x_{m n}\right)-L, \frac{t}{2}\right)<s \diamond s<\epsilon,
$$

which is impossible. Hence $Q \subset P$. Thus in both cases we conclude that $Q \subset P$. Therefore $Q \in I$. Hence $x$ is $I_{2}$ Cauchy with respect to the intuitionistic fuzzy norm $(\phi, \psi)$.

Contrarily, suppose that $x=\left(x_{i j}\right)$ is $I_{2}$-Cauchy but not $I_{2}$-convergent with respect to the intuitionistic fuzzy norm $(\phi, \psi)$. Then there exist $p$ and $q$ such that

$$
P(\epsilon, t)=\left\{(i, j) \in \mathbb{N} \times \mathbb{N}: \phi\left(B\left(x_{i j}\right)-B\left(x_{p q}\right), t\right) \leq 1-\epsilon \text { or } \psi\left(B\left(x_{i j}\right)-B\left(x_{p q}\right), t\right) \geq s\right\} \in I_{2},
$$

and

$$
Q(\epsilon, t)=\left\{(i, j) \in \mathbb{N} \times \mathbb{N}: \phi\left(B\left(x_{i j}\right)-L, \frac{t}{2}\right)>1-\epsilon \text { or } \psi\left(B\left(x_{i j}\right)-L, \frac{t}{2}\right)<\epsilon\right\} \in I_{2} .
$$

Equivalently, $Q^{c}(\epsilon, t) \in \mathcal{F}\left(I_{2}\right)$. Since

$$
\phi\left(B\left(x_{i j}\right)-B\left(x_{m n}\right), t\right) \geq 2 \phi\left(B\left(x_{i j}\right)-L, \frac{t}{2}\right)>1-\epsilon
$$

and

$$
\psi\left(B\left(x_{j k}\right)-B\left(x_{p q}\right), t\right) \leq 2 \phi\left(B\left(x_{i j}\right)-L, \frac{t}{2}\right)<\epsilon,
$$

if $\phi\left(B\left(x_{i j}\right)-L, \frac{t}{2}\right)>\frac{1-\epsilon}{2}$ and $\psi\left(B\left(x_{i j}\right)-L, \frac{t}{2}\right)<\frac{\epsilon}{2}$, respectively, we obtain $P^{c}(\epsilon, t) \in I_{2}$ and so $P(\epsilon, t) \in \mathcal{F}\left(I_{2}\right)$, which contradicts our assumption. 
Theorem 3.7 Suppose ${ }_{2} S_{(\phi, \psi)}^{I_{2}}(B)$ be an intuitionistic fuzzy normed space such that ${ }_{2} S_{(\phi, \psi)}^{I_{2}}(B)$ has a convergent subsequence. Then ${ }_{2} S_{(\phi, \psi)}^{I_{2}}(B)$ is complete.

Proof Let $\left(x_{i_{m j n}}\right)$ be a subsequence of Cauchy sequence $\left(x_{i j}\right)$ that converges to $x$. We show that $\left(x_{i j}\right) \rightarrow x$ as $(i, j) \rightarrow \infty$. Let $t>0$ and $\epsilon \in(0,1)$. Choose $s \in(0,1)$ in a such way that $s \diamond s \leq \epsilon$ and $(1-s) *(1-s) \geq 1-\epsilon$. Since $\left(x_{i j}\right)$ is a Cauchy sequence, $\exists N_{0} \in \mathbb{N}$ such that $\phi\left(B\left(x_{i j}\right)-B\left(x_{p q}\right), \frac{t}{2}\right)>1-s$ and $\psi\left(B\left(x_{i j}\right)-B\left(x_{p q}\right), \frac{t}{2}\right)<s$, for all $i, j, p$ and $q \geq N_{0}$. Since $\left(x_{m_{i} n_{j}}\right) \rightarrow x$, there is positive integer $i_{k}, j_{l}>N_{0}$ such that $\phi\left(B\left(x_{i_{k} j_{l}}\right)-B(x), \frac{t}{2}\right)>1-s$ and $\psi\left(B\left(x_{i_{k} j_{l}}\right)-B(x), \frac{t}{2}\right)<s$. Then, if $i, j \geq N_{0}$,

$$
\begin{aligned}
\phi\left(B\left(x_{i j}\right)-B(x), t\right) & \geq \phi\left(B\left(x_{i j}\right)-B\left(x_{i_{k} j l}\right), \frac{t}{2}\right) * \phi\left(B\left(x_{i_{k} l}\right)-B(x), \frac{t}{2}\right) \\
& \geq(1-s) *(1-s) \\
& \geq 1-\epsilon
\end{aligned}
$$

or

$$
\begin{aligned}
\psi\left(B\left(x_{i j}\right)-B(x), t\right) & \leq \psi\left(B\left(x_{i j}\right)-B\left(x_{i_{k} j l}\right), \frac{t}{2}\right) \diamond \psi\left(B\left(x_{i_{k j} l}\right)-B(x), \frac{t}{2}\right) \\
& \leq s \diamond s \\
& \leq \epsilon .
\end{aligned}
$$

Since $B$ is a bounded linear operator, $x_{i j} \rightarrow x$ as $(i, j) \rightarrow \infty$. Hence ${ }_{2} S_{(\phi, \psi)}^{I_{2}}(B)$ is complete.

\section{Conclusion}

The concept of intuitionistic fuzzy convergence of sequences has been studied by numerous researchers. In the present work, we introduced a more general type of convergence of sequence spaces, namely the intuitionistic fuzzy ideal convergence of double sequence spaces defined by a bounded linear operator. We investigated some algebraic and topological properties on these spaces. Furthermore, we contributed new tools to work with the convergence problems of sequences in the intuitionistic fuzzy settings, occurring in various fields of science and engineering.

\section{Acknowledgements}

We would like to thank reviewer for his valuable suggestions and comments which were very helpful in improving the manuscript.

\section{Funding}

This work is financially supported by Mohd Faisal Khan, Assistant Professor in Department of Mathematics, Saudi Electronic University, Riyadh, -602002, Saudi Arabia.

Availability of data and materials

Not applicable.

Competing interests

The authors declare that they have no competing interests. 


\section{Author details}

${ }^{1}$ Department of Mathematics, Aligarh Muslim University, Aligarh, India. ${ }^{2}$ Department of Mathematics, Saudi Electronic University, Riyadh, Saudi Arabia.

\section{Publisher's Note}

Springer Nature remains neutral with regard to jurisdictional claims in published maps and institutional affiliations.

Received: 3 May 2019 Accepted: 20 August 2019 Published online: 03 September 2019

\section{References}

1. Amini, M., Saadati, R.: Topics in fuzzy metric space. J. Fuzzy Math. 4, 765-768 (2003)

2. Atanassov, K.: Intuitionistic fuzzy sets. Fuzzy Sets Syst. 20, 87-96 (1986)

3. Das, P., Kostyrko, P., Wilczynski, W., Malik, P.: / and /*-convergence of double sequences. Math. Slovaca 58, 605-620 (2008)

4. Fast, H.: Sur la convergence statistique. Colloq. Math. 2, 241-244 (1951)

5. George, A., Veeramani, P.V.: On some result in fuzzy metric space. Fuzzy Sets Syst. 64, 395-399 (1994)

6. Goguen, J.A.: L-fuzzy sets. J. Math. Anal. Appl. 18, 145-174 (1967)

7. Gregori, V., Romaguera, S., Veeramani, P.V.: A note on intuitionistic fuzzy metric space. Chaos Solitons Fractals 28 , 902-905 (2006)

8. Khan, V.A., Alshlool, K.M.A.S., Abdullah, S.A.A.: Space of ideal convergent sequences of bounded linear operator. Numer. Funct. Anal. Optim. 39(12), 1278-1290 (2018)

9. Khan, V.A., Ebadullah, K.: On some new l-convergent sequence space. Math. Æterna 3(2), 151-159 (2013)

10. Khan, V.A., Ebadullah, K., Rababah, R.K.A.: Intuitionistic fuzzy Zweier I-convergent sequence spaces. Funct. Anal. Theory, Methods Appl. 1, 1-7 (2015)

11. Khan, V.A., Yasmeen, Fatima, H., Altaf, H., Lohani, Q.M.D.: Intuitionistic fuzzy l-convergent sequence spaces defined by compact operator. Cogent Math. 3, 1267904 (2016)

12. Kostyrko, P., Salat, T., Wilczynski, W.: I-convergence. Real Anal. Exch. 26(2), 669-686 (2000)

13. Kreyszig, E.: Introductory Functional Analysis with Application. Wiley, New York (1978)

14. Kumar, V., Kumar, K.: On the / and /*-convergence of sequence in fuzzy normed spaces. Adv. Fuzzy Sets Syst. 3(3), 341-365 (2008)

15. Mursaleen, M., Alotaibi, A.: On I-convergence in random 2-normed spaces. Math. Slovaca 61(6), $933-940$ (2011)

16. Mursaleen, M., Mohiuddin, S.A.: Statistical convergence of double sequences in intuitionistic fuzzy normed spaces. Chaos Solitons Fractals 41, 2414-2421 (2009)

17. Mursaleen, M., Mohiuddin, S.A.: On the ideal convergence of double sequences in probabilistic normed spaces. Mathem. Rep. 12(64)(4), 359-371 (2010)

18. Mursaleen, M., Mohiuddin, S.A.: On the ideal convergence in probabilistic normed spaces. Math. Slovaca 62,49-62 (2012)

19. Mursaleen, M., Mohiuddin, S.A., Edely, O.H.H.: On the ideal convergence of double sequences in intuitionistic fuzzy normed spaces. Comput. Math. Appl. 59,603-611 (2010)

20. Park, J.H.: Intuitionistic fuzzy metric spaces. Chaos Solitons Fractals 22, 1039-1046 (2004)

21. Saadati, R., Park, J.H.: On the intuitionistic fuzzy topological spaces. Chaos Solitons Fractals 27, 331-344 (2006)

22. Šalát, T., Tripathy, B.C., Ziman, M.: On some properties of I-convergence. Tatra Mt. Math. Publ. 28, 279-286 (2004)

23. Šalát, T., Tripathy, B.C., Ziman, M.: On I-convergence field. Ital. J. Pure Appl. Math. 17, 45-54 (2005)

24. Samanta, T.K., Jebril, I.H.: Finite dimensional intuitionistic fuzzy normed linear space. Int. J. Open Probl. Comput. Sci. Math. 2(4), 574-591 (2009)

25. Tripathy, B.C., Hazarika, B.: Paranorm I-convergent sequence spaces. Math. Slovaca 59(4), 485-494 (2009)

26. Tripathy, B.K., Tripathy, B.C.: On I-convergent double sequences. Soochow J. Math. 31(4), 549-560 (2005)

27. Zadeh, L.A.: Fuzzy sets. Inf. Control 8, 338-353 (1965)

\section{Submit your manuscript to a SpringerOpen ${ }^{\odot}$ journal and benefit from:}

- Convenient online submission

- Rigorous peer review

- Open access: articles freely available online

- High visibility within the field

- Retaining the copyright to your article

Submit your next manuscript at $\boldsymbol{s p r i n g e r o p e n . c o m ~}$ 